\title{
Anti-LAG-3 Monoclonal Antibody REGN3767
}

National Cancer Institute

\section{Source}

National Cancer Institute. Anti-LAG-3 Monoclonal Antibody REGN3767. NCI Thesaurus.

Code C132339.

A monoclonal antibody directed against the inhibitory receptor lymphocyte activation gene-3 protein (LAG-3; LAG3; CD223), with potential immune checkpoint inhibitory and antineoplastic activities. Upon administration, the anti-LAG-3 monoclonal antibody REGN3767 binds to LAG-3 expressed by tumor infiltrating lymphocytes (TILs) and blocks LAG-3 binding to tumor cells expressing major histocompatibility complex (MHC) class II molecules. This may activate antigen-specific T-lymphocytes and enhance cytotoxic Tlymphocyte (CTL)-mediated tumor cell lysis, which leads to a reduction in tumor growth. LAG-3, a member of the immunoglobulin superfamily (IgSF), is expressed on various immune cells; its expression on TILs is associated with tumor-mediated immune suppression and the negative regulation of both cellular proliferation and $\mathrm{T}$-cell activation. 